University of Nebraska - Lincoln

DigitalCommons@University of Nebraska - Lincoln

$9-2002$

\title{
Closed-form solution for a mode-III interfacial edge crack between two bonded dissimilar elastic strips
}

Xiangfa Wu

Department of Engineering Mechanics, University of Nebraska-Lincoln, xfwu@unlserve.unl.edu

Yuris A. Dzenis

University of Nebraska - Lincoln, ydzenis@unl.edu

Follow this and additional works at: https://digitalcommons.unl.edu/engineeringmechanicsfacpub

Part of the Mechanical Engineering Commons

Wu, Xiangfa and Dzenis, Yuris A., "Closed-form solution for a mode-III interfacial edge crack between two bonded dissimilar elastic strips" (2002). Faculty Publications from the Department of Engineering Mechanics. 33.

https://digitalcommons.unl.edu/engineeringmechanicsfacpub/33

This Article is brought to you for free and open access by the Mechanical \& Materials Engineering, Department of at DigitalCommons@University of Nebraska - Lincoln. It has been accepted for inclusion in Faculty Publications from the Department of Engineering Mechanics by an authorized administrator of DigitalCommons@University of Nebraska - Lincoln. 
Published in Mechanics Research Communications 29:5 (September-October 2002), pp. 407-412; doi 10.1016/S0093-6413(02)00317-8 Copyright @ 2002 Elsevier Science Ltd.

Used by permission. http://www.elsevier.com/wps/product/cws home/374

Submitted January 8, 2002; published online September 4, 2002.

\title{
Closed-form solution for a mode-III interfacial edge crack between two bonded dissimilar elastic strips
}

\author{
Xiang-Fa Wu and Yuris A. Dzenis \\ Department of Engineering Mechanics, Center for Materials Research and Analysis, \\ University of Nebraska-Lincoln, Lincoln, NE 68588-0526, USA \\ Corresponding author - Xiang-Fa Wu, email xfwu@unlserve.unl.edu
}

\begin{abstract}
A closed-form solution is obtained for the problem of a mode-III interfacial edge crack between two bonded semi-infinite dissimilar elastic strips. A general out-of-plane displacement potential for the crack interacting with a screw dislocation or a line force is constructed using conformal mapping technique and existing dislocation solutions. Based on this displacement potential, the stress intensity factor $\left(\mathrm{SIF}, K_{\mathrm{III}}\right)$ and the energy release rate $\left(E R R, G_{\mathrm{III}}\right)$ for the interfacial edge crack are obtained explicitly. It is shown that, in the limiting special cases, the obtained results coincide with the results available in the literature. The present solution can be used as the Green's function to analyze interfacial edge cracks subjected to arbitrary anti-plane loadings. As an example, a formula is derived correcting the beam theory used in evaluation of SIF $\left(K_{\mathrm{III}}\right)$ and ERR $\left(G_{\mathrm{III}}\right)$ of bimaterials in the double cantilever beam (DCB) test configuration.
\end{abstract}

Keywords: interfacial crack, edge crack, screw dislocation, stress intensity factor, strip, bimaterial, double cantilever beam

\section{Introduction}

Models describing interactions of cracks and dislocations play an important role in fracture mechanics as their solutions can serve as fundamental solutions for cracks with more complicated geometry and loading configurations (Rice and Thomson, 1974; Ohr, 1985; Weertman, 1996). A number of investigators have contributed significantly to this subject (see Thomson, 1986; Suo, 1989; Suo, 1990; and references). Finite or semi-infinite cracks in an infinite medium were usually considered. However, in practice, edge cracks are likely to occur in composite laminates and bonded structures, such as adhesive joints. The main failure mode of these materials is edge delamination or edge debonding between the laminas or dissimilar components (Jones, 1999). Recently, interfacial edge crack problems attracted considerable attention. Shiue et al. (1989) studied screw dislocations interacting with an interfacial edge crack between two bonded dissimilar isotropic quarter-planes. Choi et al. (1994) considered an interfacial edge crack between two bonded dissimilar orthotropic quar- 
ter-planes under anti-plane shear. Lee and Earmme (2000) discussed an interfacial edge crack in an anisotropic bimaterial (half-plane) under anti-plane singularity.

The conformal mapping technique was extensively utilized for finding explicit solutions in fracture mechanics. Based on this technique, a complicated boundary value problem can be reduced to a Riemann-Hilbert problem along a line or an arc. Suo (1990) provided a general complex potential for two bonded dissimilar anisotropic half-planes with a semi-infinite interfacial crack interacting with an anti-plane singularity (a screw dislocation or a line-force). Using this potential in conjunction with a proper conformal mapping, the interfacial edge crack problem can be solved explicitly for more complicated, practically important geometries.

Most interfacial edge crack problems analyzed in the literature to date considered cracks between two bonded quarter-planes. The objective of this work is to study the anti-plane problem and to determine the stress intensity factor $\left(\mathrm{SIF}, K_{\mathrm{III}}\right)$ and the energy release rate $\left(\mathrm{ERR}, \mathrm{G}_{\mathrm{III}}\right)$ for an interfacial edge crack between two bonded dissimilar elastic strips. A closed-form solution is obtained by using the conformal mapping technique and the known potential for the simpler crack geometry. Several special cases are considered and the results are shown to be consistent with the literature data in the limiting configurations. As a special outcome, a formula is provided correcting the beam theory used in evaluation of SIF and ERR of bimaterials in the double cantilever beam (DCB) configuration. The latter test configuration is used in fracture testing of composites and bonded structures.

\section{Formulation and solution procedure}

The out-of-plane displacement and shear stresses in an elastic body subjected to anti-plane deformation can be expressed in terms of an analytic function $\varphi(\zeta)$ as follows:

$$
u_{3}(\xi, \eta)=-\frac{2}{\mu} \operatorname{Im}[\varphi(\zeta)], \quad \sigma_{23}(\xi, \eta)=-2 \operatorname{Re}\left[\varphi^{\prime}(\zeta)\right], \quad \sigma_{13}(\xi, \eta)=2 \operatorname{Re}\left[i \varphi^{\prime}(\zeta)\right]
$$

where prime (') denotes the derivative with respect to $\zeta=\xi+i \eta$, and $\mu$ is the material shear modulus.

Let us first consider the out-of-plane displacement potential of the two bonded dissimilar elastic half-planes with a semi-infinite interfacial crack subjected to an anti-plane singularity, as shown in Figure 1(b). Without loss of generality, the singularity can be assumed to be located in the lower half-plane at $\zeta_{0}=\xi_{0}+\mathrm{i} \eta_{0}\left(\eta_{0}<0\right)$, where $\xi$ and $\eta$ are coordinates with the origin of the coordinate system located at the crack tip. The complex potential of this problem can be obtained as a special case of the problem discussed by Suo (1990) as follows:

$$
\begin{aligned}
\varphi^{\prime}(\zeta) & =\frac{2 \mu_{1}}{\mu_{1}+\mu_{2}} \frac{q}{\zeta-\zeta_{0}}+\varphi_{0}^{\prime}, \quad(\eta>0) \\
\varphi^{\prime}(\zeta) & =\frac{q}{\zeta-\zeta_{0}}+\bar{q} \frac{\mu_{1}-\mu_{2}}{\mu_{1}+\mu_{2}} \frac{1}{\zeta-\bar{\zeta}_{0}}+\varphi_{0}^{\prime}, \quad(\eta<0)
\end{aligned}
$$

where

$$
\varphi_{0}^{\prime}(\zeta)=\frac{\mu_{1}}{\mu_{1}+\mu_{2}}\left\{\frac{q}{\zeta-\zeta_{0}}\left[\left(\frac{\zeta_{0}}{\zeta}\right)^{1 / 2}-1\right]+\frac{\bar{q}}{\zeta-\bar{\zeta}_{0}}\left[\left(\frac{\bar{\zeta}}{\zeta}\right)^{1 / 2}-1\right]\right\} .
$$

Subscripts 1 and 2 in (2)-(4) denote material properties; overbar $\left(^{-}\right)$denotes the complex conjugate; and the quantity $q$ is defined as 


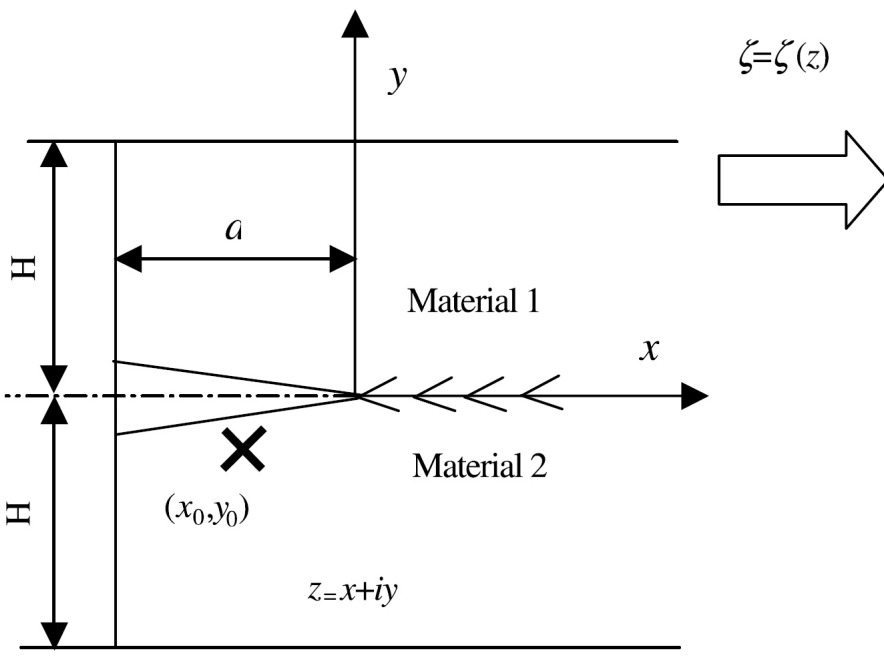

(a)

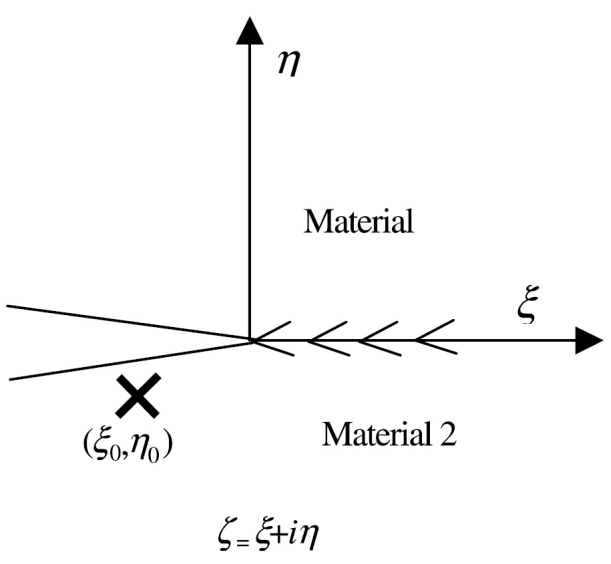

(b)

Figure 1. A cracked bimaterial under anti-plane singularity: (a) an edge-cracked bimaterial strip (z-plane); (b) a semi-infinite interfacial crack between two bonded dissimilar elastic half-planes ( $\zeta$-plane).

$$
q=-\frac{b \mu_{2}}{4 \pi}+\mathrm{i} \frac{p}{4 \pi}
$$

where $b$ is the Burgers vector of the screw dislocation and $p$ is the line-force.

The SIF and ERR can be evaluated using the following relationships:

$$
K_{\mathrm{III}}=\lim _{\xi \rightarrow 0}-2 \sqrt{2 \pi \xi} \varphi^{\prime}(\xi), \quad G_{\mathrm{III}}=\frac{1}{4}\left(\frac{1}{\mu_{1}}+\frac{1}{\mu_{2}}\right) K_{\mathrm{III}}^{2}
$$

For the particular case studied, the following formula is derived:

$$
K_{\mathrm{III}}=2 \sqrt{2 \pi} \frac{\mu_{1}}{\mu_{1}+\mu_{2}}\left[\frac{q}{\left(\zeta_{0}\right)^{1 / 2}}+\frac{\bar{q}}{(\bar{\zeta})^{1 / 2}}\right] .
$$

Let us now consider an interfacial edge crack between two bonded semi-infinite dissimilar elastic strips subjected to an anti-plane singularity (a screw dislocation or a line-force) on the lower strip at $z_{0}=x_{0}+\mathrm{i} y_{0}\left(y_{0}<0\right)$, as shown in Figure 1(a). Here $a$ and $H$ denote the edge crack length and the strip width, respectively. The conformal mapping,

$$
\zeta=\frac{g^{2}(z+a)}{g^{2}(a)}-1
$$

with

$$
g(z)=\sinh \left(\frac{\pi z}{2 H}\right),
$$

will map the strips onto half-planes with a semi-infinite cut along the negative $\xi$-axis, as shown in Figure 1(b). Substitution of (8) and (9) into (2)-(4) leads to the complex displacement potential for the bonded strips. Its main contributor (4) is expressed as 


$$
\begin{aligned}
\varphi_{0}^{\prime}(z)= & \frac{\mu_{1}}{\mu_{1}+\mu_{2}} \frac{\pi}{2 H} g[2(z+a)]\left\{\frac{q}{g^{2}(z+a)-g^{2}\left(z_{0}+a\right)}\left\{\left[\frac{g^{2}\left(z_{0}+a\right)-g^{2}(a)}{g^{2}(z+a)-g^{2}(a)}\right]^{1 / 2}-1\right\}\right. \\
& \left.+\frac{\bar{q}}{g^{2}(z+a)-\overline{g^{2}\left(z_{0}+a\right)}}\left\{\left[\frac{g^{2}\left(z_{0}+a\right)-g^{2}(a)}{g^{2}(z+a)-g^{2}(a)}\right]^{1 / 2}-1\right\}\right\},
\end{aligned}
$$

where $q$ is the singularity quantity defined in (5).

Substitution of (2)-(5) and (10) into (6) yields the following expression for the SIF:

$$
K_{\mathrm{III}}=\frac{4 \sqrt{2} \pi}{\sqrt{\pi a}} \frac{\mu_{1}}{\mu_{1}+\mu_{2}} \sqrt{\frac{\pi a}{H} \sinh \left(\frac{\pi a}{H}\right)} \operatorname{Re}\left\{-i q / \sqrt{\cosh \left(\frac{\pi a}{H}\right)-\cosh \left[\frac{\pi\left(z_{0}+a\right)}{H}\right]}\right\} .
$$

Here, $\operatorname{Re}()$ is the real part of an analytic function. The corresponding ERR can be obtained by inserting (11) into (6).

If the singularity is located in the upper strip, the SIF and ERR can be derived by analogy, or can simply be obtained by exchanging the material subscripts in (11). The relation (11) can be used as the Green's function to determine the SIF and ERR for the edge-cracked bimaterial strips subjected to arbitrary anti-plane loadings applied on the crack or edge surfaces or in the interior of the strips.

\section{Examples}

Here we obtain the SIF and ERR solutions for two special cases particularly useful in fracture mechanics.

\subsection{Edge crack with anti-plane forces applied on crack surfaces}

Setting $q=\mathrm{i} P /(4 \pi)$ and $z_{0}=-b-0 \mathrm{i}$ in (11), we obtain the SIF for the line-force $P$ located on the lower crack surface at a distance $b$ behind the crack tip:

$$
K_{\mathrm{III}}=\frac{\sqrt{2} P}{\sqrt{\pi a}} \frac{\mu_{1}}{\mu_{1}+\mu_{2}} \sqrt{\frac{\pi a}{H} \sinh \left(\frac{\pi a}{H}\right)} / \sqrt{\cosh \left(\frac{\pi a}{H}\right)-\cosh \left[\frac{\pi(a-b)}{H}\right]} .
$$

Further, by letting $H \rightarrow \infty$ in (12), we can extract the SIF solution for the edge crack between two bonded elastic quarter-planes,

$$
K_{\mathrm{III}}=\frac{2 P}{\sqrt{\pi a}} \frac{\mu_{1}}{\mu_{1}+\mu_{2}} \frac{a}{\sqrt{a^{2}-(a-b)^{2}}} .
$$

The latter expression is in agreement with the solution obtained by Choi et al. (1994) and Lee and Earmme (2000). Alternatively, the result (13) can be derived straightforwardly by letting $H \rightarrow \infty$ in (9) and by using the mapping function $\zeta=(z / a+1)^{2}-1$.

Now consider a pair of line-forces $P$ acting on the lower and upper crack surfaces at $z_{0}=-b \pm 0$ i. Here $z_{0}=-b+0 \mathrm{i}$ and $z_{0}=-b-0 \mathrm{i}$ denote the points in the upper and lower crack surfaces, respectively. The corresponding SIF can be obtained as 


$$
K_{\mathrm{III}}=\frac{\sqrt{2} P}{\sqrt{\pi a}} \sqrt{\frac{\pi a}{H} \sinh \left(\frac{\pi a}{H}\right)} / \sqrt{\cosh \left(\frac{\pi a}{H}\right)-\cosh \left[\frac{\pi(a-b)}{H}\right]} .
$$

By letting $a \rightarrow \infty$ in (14), we can get the SIF for a semi-infinite crack between two bonded strips:

$$
K_{\mathrm{III}}=P \sqrt{\frac{2}{H}} / \sqrt{1-\exp \left(-\frac{\pi b}{H}\right)}
$$

which coincides with the result in the literature (Sih, 1973) except for a constant $\sqrt{\pi}$ due to the different SIF definition.

Furthermore, integration of (14) with respect to $b$ in the interval $[0, a]$ yields the SIF for the edge crack loaded with the uniform forces $p$ acting on the crack surfaces:

$$
K_{\mathrm{III}}=p \sqrt{\pi a} \frac{2}{\pi} \sqrt{\frac{2 H}{\pi a} \tanh \left(\frac{\pi a}{2 H}\right)} K\left[\tanh \left(\frac{\pi a}{2 H}\right)\right],
$$

where $K()$ is the complete elliptic integral of the first kind. The result (16) is equivalent to the known solution for the finite interfacial crack of length $2 a$ embedded between two bonded infinite strips and loaded with uniform anti-plane shear applied on the crack surfaces. The latter problem was solved by Singh et al. (1981) and Li (2001) using dual integral equations.

\subsection{Edge crack with anti-plane forces applied on edge surfaces}

Now let us consider a pair of line-forces, $P$ acting symmetrically on the edge surfaces at $z_{0}=-a \pm$ $\mathrm{i} h$. By setting $q=\mathrm{i} P /(4 \pi)$ and $z_{0}=-a \pm \mathrm{i} h$ in (11), we obtain the corresponding SIF:

$$
K_{\mathrm{III}}=\frac{\sqrt{2} P}{\sqrt{\pi a}} \sqrt{\frac{\pi a}{H} \sinh \left(\frac{\pi a}{H}\right)} / \sqrt{\cosh \left(\frac{\pi a}{H}\right)-\cos \left(\frac{\pi h}{H}\right)} .
$$

Consequently, integration of (17) with respect to $h$ in the interval $[0, H]$ yields the SIF for the crack loaded by uniform forces $p$ acting on the edge surfaces:

$$
K_{\text {III }}=p \sqrt{\pi a} \frac{2}{\pi} \sqrt{\frac{H}{\pi a} \sinh \left(\frac{\pi a}{H}\right)} F\left\{\cosh \left(\frac{\pi a}{2 H}\right), \arcsin \left[\cosh ^{-1}\left(\frac{\pi a}{2 H}\right)\right]\right\},
$$

where $F($ ) is the Legendre's elliptic integral of the first kind.

The ERR for all cases discussed above can be easily obtained by using the relationship between the ERR and SIF (6).

The relations (17) and (18) can be used to correct the beam theory that is often utilized for data reduction in experimental evaluation of SIF $\left(K_{\mathrm{III}}\right)$ and ERR $\left(G_{\mathrm{III}}\right)$ in the DCB test configuration.

\section{Acknowledgments}

Partial support of this work by the U.S. Army Research Office and the U.S. Air Force Office of Scientific Research is gratefully acknowledged. 


\section{References}

Choi et al., 1994 - S. R. Choi, C. H. Chong and Y. S. Chai, Interfacial edge crack in two bonded dissimilar orthotropic quarter planes under antiplane shear. Int. J. Fract. 67 (1994), pp. 143-150.

Jones, 1999 - M. R. Jones, Mechanics of Composite Materials (2nd edition), Taylor \& Francis, Philadelphia (1999).

Lee and Earmme, 2000 - K. W. Lee and Y. Y. Earmme, An interfacial edge crack in anisotropic bimaterial under anti-plane singularity. Int. J. Fract. 104 (2000), pp. 13-22.

Li, 2001 - X. F. Li, Closed-form solution for a mode-III interface crack between two bonded dissimilar elastic layers. Int. J. Fract. 109 (2001), pp. L3-L8.

Ohr, 1985 - S. M. Ohr, An electron microscope study of crack tip deformation and its impact on the dislocation theory of fracture. Mater. Sci. Eng. 72 (1985), pp. 1-35.

Rice and Thomson, 1974 - J. R. Rice and R. Thomson, Ductile versus brittle behavior of crystals. Philos. Mag. 29 (1974), pp. 73-97.

Shiue et al., 1989 - S. T. Shiue, C. T. Hu and S. Lee, Elastic interaction between screw dislocations and a welded surface crack in composite materials. Eng. Fract. Mech. 335 (1989), pp. 697-706.

Sih, 1973 - G. C. Sih, Handbook of Stress Intensity Factors, Lehigh University, Bethlehem, Pennsylvania (1973).

Singh et al., 1981 - B. M. Singh, T. B. Moodie, and J. B. Haddow, Closed-form solutions for finite length crack moving in a strip under antiplane shear stress. Acta Mech. 38 (1981), pp. 99-109.

Suo, 1989 - Z. Suo, Singularities interacting with interfaces and cracks. Int. J. Solids Struct. 2510 (1989), pp. $1133-1142$.

Suo, 1990 - Z. Suo, Singularities, interfaces and cracks in dissimilar anisotropic media. Proc. R. Soc. Lond. A427 (1990), pp. 331-358.

Thomson, 1986 - R. Thomson, Physics of fracture. Solid State Phys. 39 (1986), pp. 1-129.

Weertman, 1996 - J. Weertman, Dislocation Based Fracture Mechanics, World Scientific Publishing, Singapore (1996). 